\title{
Dos décadas de estudios del Paisaje Lingüístico: enfoques teórico-metodológicos y nuevos desafíos en la investigación
}

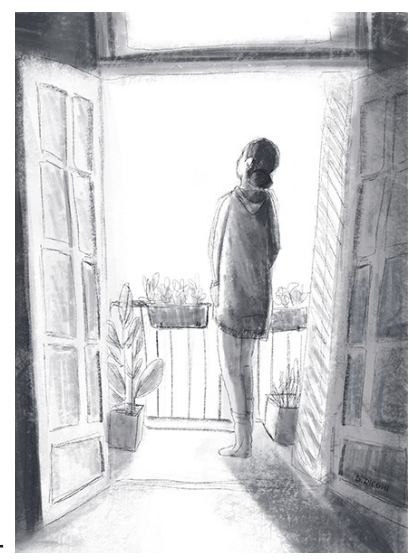

Adil Moustaoui Srhir

Universidad Complutense de Madrid, España

adilmous@ucm.es

Trabajo recibido el 10 de abril de 2019 y aprobado el 12 de junio de 2019.

\section{Resumen}

En los últimos 20 años, el Paisaje Lingüístico (PL en adelante) -linguistic landscape en inglés- se convirtió en enfoque de investigación sociolingüística cuyo foco de análisis es el uso de las lenguas en el espacio. El PL como método de análisis de datos en la sociolingüística urbana ha llegado a abarcar varios objetos de estudio, pudiendo responder a una serie de desafíos para el análisis del multilingüismo y la multimodalidad en la comunicación en relación con el espacio. Su desarrollo en las últimas décadas ayudó en su consolidación posteriormente como una línea de investigación sobre el uso de la lengua en la construcción del espacio, y por tanto, como objeto de estudio potencialmente atractivo para la lingüística aplicada y la sociología de la lengua y del espacio. El objetivo de este artículo es ofrecer pues un recorrido por los principales enfoques teórico-metodológicos que se han desarrollado a lo largo de estas dos últimas décadas para el análisis y el estudio del PL. Dichos enfoques se han caracterizado por ser amplios, variados y diversificados, donde incluso otros cuestionaban la propia categoría de PL como objeto de análisis en el campo de la sociolingüística. Del mismo modo, mi intención es intentar desarrollar un balance, un esbozo de contraste y un desarrollo histórico de estos enfoques con una visión relativamente amplia que refleje las distintas categorías y herramientas de análisis empleadas en los estudios del PL, haciendo un especial énfasis en la investigación producida durante la última década.

\section{Two decades in Linguistic Landscape studies: theoretical and methodological approaches and new challenges for research}

\author{
Abstract \\ In the last 20 years, the Linguistic Landscape (PL onwards) became a \\ sociolinguistic research focus on the use of languages in space. The LL as \\ a method of data analysis in urban sociolinguistics has come to encompass
}

Palabras clave

Paisaje Lingüístico espacio lengua

sociolingüística urbana comunicación multimodal
Keywords

Linguistic landscape space language

sign

urban sociolinguistics multimodal communication 
several objects of study, being able to respond to a series of challenges for the analysis of multilingualism and multimodality in relation to space. The development of LL in the last decades helped in its consolidation later as a line of research on the use of language in the construction of space, and therefore as an object of study potentially attractive for applied linguistics. So, the objective of this article is to offer an overview of the main theoretical-methodological approaches that have been developed over the last two decades for the analysis and study of LL. These approaches have been characterized by being broad, varied and diversified, where even other approaches questioned the LL category itself as an object of sociolinguistic analysis. Likewise, my intention is to try to develop a balance, outline of contrast and historical development of these approaches with a relatively broad vision that reflects their diversity and the different tools of analysis, emphasizing the research produced over the last decade.

\section{Duas décadas em estudos da Paisagem Linguística: abordagens teóricas e metodológicas e novos desafios em pesquisa}

\section{Resumo}

Nos últimos 20 anos, a Paisagem Linguística (linguistic landscape em inglês e PL daqui em diante) tornou-se um foco de pesquisa sociolinguística, cujo objeto de análise é o uso das línguas na construção do espaço. Como método de análise de dados na sociolinguística urbana, a PL passou a abranger vários objetos de estudo, que ajudam a dar resposta a uma série de desafios para a análise do multilinguismo e da multimodalidade na comunicação em articulação com o espaço. O desenvolvimento das últimas décadas ajudou a consolidar uma linha de pesquisa sobre o uso linguístico na construção do espaço, logo, potencialmente atrativo para a linguística aplicada, para a sociologia da linguagem e para as abordagens sociais de espacialidade. $\mathrm{O}$ objetivo deste artigo é oferecer um roteiro das principais abordagens teórico-metodológicas desenvolvidas ao longo das duas últimas décadas para a análise e estudo da PL. Se algumas dessas abordagens foram amplas, variadas e diversificadas, outras houve que questionaram a própria categoria de LP como objeto de análise no campo da sociolinguística. Por essa razão, faço um esboço deste contraste, contextualizando as abordagens na sua história. Tal permite uma visão relativamente ampla sobre as diferentes ferramentas e categorias de análise utilizadas em PL, com especial ênfase na pesquisa produzida durante a última década.

\section{Introducción}

Históricamente, gran parte de las investigaciones en sociología de la lengua se centraron en el análisis de categorías y aspectos que tienen que ver en su mayoría con la modalidad y el discurso oral en la interacción. Esta atención y este interés prestados a las interacciones en su modalidad oral excluyeron en cierto modo el análisis del PL, en particular, y prácticas de la escritura en el espacio, en general, de los estudios sociolingüísticos. Esta falta de interés por la dimensión espacial de las prácticas lingüísticas, no se dio únicamente en la sociolingüística occidental, sino también en otras sociolingüísticas periféricas, producidas desde otros lugares y continentes. Sin embargo, las nuevas líneas de investigación sociolingüística y semiótica

\section{Palavras-chave}

Paisagem Linguística espaço lingua signo sociolinguística urbana multimodalidade na comunicação 
demostraron perfectamente que el régimen de uso de las lenguas en el espacio, independientemente de su dimensión semiótica, es también un conjunto de prácticas socio-comunicativas insertadas en unas dinámicas y unos procesos muy complejos que requieren nuevos enfoques sociolingüísticos y nuevas herramientas de análisis (Blommaert 2013a, Lillis y Mackinney 2013).

La aparición del concepto de Paisaje Lingüístico (PL en adelante) en un artículo publicado por Landry y Bourhis (1997) fue una aportación empírica al campo de la sociolingüística urbana. En este artículo, los autores, definieron PL como el conjunto de textos públicamente visibles en cualquier lengua escrita, a saber, letreros, carteles, pancartas, señales, graffiti y todo tipo de inscripciones, tanto las elaboradas profesionalmente como de manera improvisada, presentes en el espacio público y privado. Es a partir de esta publicación que se abrió un nuevo campo de investigación en la sociolingüística que estaba ausente hasta la fecha, a saber, Los estudios del Paisaje Lingüístico (EPL de aquí en adelante) o (Linguistic Landscape Studies/ LLS). Esta nueva línea de investigación, se centra principalmente en la exploración de las representaciones de la(s) lengua(s) en el espacio público. Su objeto de estudio puede ser cualquier práctica visible del lenguaje escrito (un signo), así como la interacción a través del propio signo en el espacio. Es una línea de investigación altamente interdisciplinar que usa una extensa escala de teorías, metodologías y disciplinas, como la política lingüística, la semiótica, los estudios de literacidad, la antropología, la sociología, la geografía social y humana, estudios de urbanismo, economía o turismo.

La necesidad pues de explorar en los EPL la relación lengua/ construcción, permite descubrir la forma en que los hablantes, los actores sociales y las comunidades lingüísticas crean, reinventan, negocian y construyen sus prácticas y usos de la lengua en un espacio y contexto histórico determinados. De la misma forma, el EPL se interesa por analizar la construcción de las relaciones sociales a través de prácticas lingüísticas en el propio espacio, ofreciendo interpretaciones sobre las dinámicas de cambio socio-políticas, económicas, urbanas, ideológicas e identitarias y el papel de la agencia de los hablantes en la transformación de los regímenes sociolingüísticos. De allí radica la trascendencia de los EPL como enfoque que aporta otra lectura y una interpretación más amplia de la dimensión lingüístico-comunicativa, social y política de la lengua, sus signos y sus prácticas en el seno del espacio. Tal como destacan Castillo y Sáez $(2013,12)$ :

\section{Los estudios del paisaje lingüístico comparten básicamente estas mismas problemáticas relativas al PL como construcción social: ¿qué es lo que estructura el uso de las lenguas en el espacio público?, ¿qué dinámicas se aprecian en esos usos?, ¿bajo qué formas, dialectales, sociolectales, dianormativas, etc. se presentan los mensajes?, [...] ¿cómo se reparten las lenguas (en) el espacio?, ¿cómo se organiza normativamente el uso de las lenguas?, ¿cuáles de ellas son centrales y periféricas?}

El crecimiento en los últimos 20 años del uso del PL como herramienta de investigación y análisis de corpus semiótico de datos, ha llegado a ser muy amplio pudiendo responder a una serie de fenómenos relacionados también con la comunicación multimodal y el multilingüismo. En los últimos cinco años también han proliferado enfoques en los EPL, en el que se ha incorporado la metodología cuantitativa, y las categorías de análisis se han ido extendiéndose, especialmente en entornos multilingües. Varios estudios de 
corte sociolingüístico-etnográfico empezaron a combinar también métodos cuantitativos para ofrecer nuevas interpretaciones a partir del análisis del PL. Del mismo modo, se ha visto como los datos y corpus que se recogen para el análisis del PL se introdujeron en estudios de carácter económico, en investigaciones en ciencias políticas y también en estudios sobre el turismo (ver Marten et al. 2012, Javorski 2010).

Es digno de mencionar también la incorporación del PL en la exploración de muchas cuestiones de carácter sociolingüístico, ideológico, pedagógicodidáctico y político en el espacio de la escuela. El hecho de que los paisajes escolares funcionan de manera similar que otros paisajes hace que estén implicados en la reproducción social de la vida cotidiana. Metodologías de análisis como school-based linguistic landscape research o schoolscapes (Brown 2012, 2005) emergieron como nuevas herramientas para explorar el entorno escolar, siendo un espacio social donde se producen, tanto discursos escritos (gráficos y signos) como orales, a través de los cuales se constituyen, reproducen y transforman las ideologías lingüísticas y se crean también Paisajes Escolares. Los estudios del Paisaje Lingüístico se introdujeron también como herramientas pedagógicas para la enseñanza de las lenguas extranjeras (Aldjem y Jou 2016), también como recurso relevante para ser integrado en la investigación-acción en el aula sobre el desarrollo de la conciencia metalingüística y el valor de las lenguas (Prego Vázquez y Zas Varela 2018), o para analizar la función del signo y del PL en entornos escolares multilingües en los trabajos de Gorter y Cenoz (2015) en el contexto del País Vasco.

Por último, señalar que no menos importante es el análisis de las ideologías lingüísticas y las representaciones socio-discursivas en los estudios sobre el PL. La conexión entre PL e ideologías demuestra cómo el régimen de uso de las lenguas y las variedades lingüísticas en el espacio está vinculado directamente con la producción de ideologías lingüísticas y actitudes que justifican modelos de construcción del espacio y de regimentación sociolingüística. Unos modelos que influyen en cómo se regula y legitima posteriormente la producción de nuevas prácticas lingüísticas en el espacio; sea público o privado, y por lo tanto son claves en la configuración de nuevos Paisajes Lingüísticos.

La amplia literatura producida hasta el momento ha hecho por lo tanto que los estudios sobre PL adquirían un lugar y una posición considerables en las dos últimas décadas en la sociolingüística y se consolidara una línea de investigación potencialmente atractiva y rica (Shohamy 2006, 2015; Barni y Bagna 2015). Como consecuencia, el PL se aborda y analiza cada vez más desde la interdisciplinariedad y con distintos métodos y enfoques, lo que permitió que surgieran nuevos conceptos más abiertos y amplios, y también nuevos componentes en el análisis del PL.

El objetivo pues de este artículo es ofrecer un recorrido por los principales enfoques teóricos y metodológicos que se han desarrollado a lo largo de estas dos últimas décadas para el análisis y el estudio del PL. Uso la palabra 'principales', porque estos enfoques se han caracterizado por ser amplios, variados y diversificados. E incluso otras escuelas han discutido la propia noción de PL como categoría de análisis sociolingüístico. Mi intención tampoco consiste en desarrollar una discusión teórico-crítica sobre dichos enfoques; más bien busco desarrollar un balance, haciendo énfasis en la última década, con una visión relativamente amplia que refleje la diversidad de los enfoques y herramientas de análisis en los estudios del PL. 


\section{Espacio, lengua y Paisaje Lingüístico}

En primer lugar, creo que conviene ubicar la noción del espacio sea urbano o de otro carácter dentro del PL y el régimen de uso de las lenguas. En la definición de espacio propuesta por Lefevbre $(1991,26)$, se le considera como una producción social y un conjunto de relaciones entre distintos elementos, considerados como objetos y productos (Lefevbre 1991, 83). De este modo y tal como señala Lefevbre (op.cit.), la construcción de nuevas relaciones sociales implica la configuración de nuevos espacios y viceversa. El espacio pues por su carácter híbrido se considera adecuado para las acciones y prácticas lingüísticas que vendrían a configurarlo y construirlo igual que su PL. El carácter doblemente apropiado del espacio permite que sea usado con finalidades comunicativas, y transformado simbólica y representativamente en la medida en que se configuren otro(s) espacio(s), visible(s), abiertos y distintos - aunque también compartidos-al tradicional espacio que podría estar bajo control del estado, las instituciones oficiales o cualquier organismo o agente social.

Por otra parte, Lefebvre introdujo el concepto del derecho a la ciudad, concebido originalmente como el derecho a la participación (a contribuir en la producción y construcción del espacio urbano) y a su posterior apropiación, es decir, usado y ocupado. Este derecho se ha convertido en una demanda fundamental de los movimientos sociales y otros actores sociales, tanto para reconfigurar los procesos de urbanización como para cuestionar, repensar y replantear el concepto de ciudadanía, tradicionalmente ligado al estado nación. Una de las manifestaciones de dichas demandas es la ocupación de los espacios urbanos, lo cual conlleva fenómenos tales como la desterritorialización (apertura) y la reterritorialización /apropiación (Martin Rojo 2014), en el que se producen nuevas prácticas discursivas y comunicativas, nuevos modelos de organización y también se generan nuevas identidades y subjetividades políticas. En los últimos años, hemos visto cómo movimientos sociales y actores de la sociedad civil han ocupado las plazas para transformarlas en espacios deliberativos, han demandado el uso público o común de bienes (de la vivienda, del transporte) o han promovido nuevos imaginarios de la ciudad para promover el cambio social. Estas prácticas de resistencia frente al orden neo-liberal y capitalista operan igualmente en diferentes escalas (local-nacional-global) en las que se generan redes ciudadanas y cosmopolitas (Moustaoui Srhir y García Agustín 2015), capaces de transformar el orden espacial, discursivo y lingüístico.

En segundo lugar, cabe subrayar que en el mismo espacio los individuos y las comunidades de práctica comunican, interactúan y generan nuevas prácticas lingüísticas, siendo el espacio perfectamente adecuado para estas mismas prácticas lingüísticas. Blommaert, Collins y Slembrouk señalan $(2005,203)$ que:

Every instance of human communication always has an intrinsic spatiality to it as well as an intrinsic temporality. Every communication event develops in some time-frame and in some space, and both, as we know, have effects on what happens and can happen. Space is part of what we understand by 'context', and context (as Gumperz (1982) and other have argued) is not a passive 'decor' but an active, agentive aspect of communication.

En tercer lugar, el espacio es único, no sólo porque consiste en un conjunto de componentes y elementos que interactúan entre sí, sino porque es también creativo, dinámico, en constante evolución y al alcance de un amplio número de personas y actores que lo construyen. 
Blommaert y Maly $(2015,192)$, en una excelente justificación del porqué la sociolingüística ha de interesarse por el espacio y el PL, afirman que los estudios sobre PL:

Can detect and interpret social change and transformation on several scale-levels, from the very rapid and immediate to the very slow and gradual ones, all gathered in a 'synchronic' space. A detailed and nuanced LLS (Linguistic Landscape Studies) can thus describe the layered, multifiliar and nonlinear nature of sociolinguistic phenomena-in other words: it opens the way to a

Una sociolingüística del espacio y de su paisaje lingüístico nos ayuda por lo tanto a comprender en palabras de Heller $(2005,322)$ "l'organisation sociale de la ville et, plus largement, du rôle de la ville dans les processus de structuration sociale".

La reconsideración por parte de la sociolingüística de los espacios urbanos, sea públicos o privados, como representaciones de procesos inseparables de factores geográficos, demográficos, históricos, socio-económicos, políticos, ideológicos y semióticos, invita a EPL y la investigación en sociolingüística urbana, en general, a tomar cartas en el asunto haciéndose participe en el análisis de estos fenómenos y siguiendo una diversidad de enfoques.

\section{Paisaje Lingüístico: temáticas y contenidos}

\subsection{Breve contextualización}

Tal como he mencionado anteriormente, y a partir de la definición de PL propuesta por Landry y Bourhis (1997), la mayoría de las investigaciones posteriores se centraron al principio en el análisis de las funciones y del papel que desempeñan las lenguas en diferentes espacios públicos y privados. Incluso, el planteamiento inicial que ofrecieron Landry y Bourhis (1997) para el estudio del PL, hacía referencia a muchos desarrollos, tanto teóricos como prácticos, que todavía hoy en día se aplican en el análisis del objeto. Pero más tarde, en Gorter (2006) la investigación sobre el PL experimentó un giro importante dado que el interés de los investigadores empezó a centrarse cada vez menos en las funciones de las variedades lingüísticas en los espacios públicos de carácter oficial o no oficial. ${ }^{1}$ Dicho interés se focalizaba en cómo las relaciones de poder y las desigualdades políticas, sociales y económicas ejercían una influencia directa sobre la construcción y la configuración del PL en las ciudades, a partir de políticas lingüísticas oficiales conscientes en un contexto de multilingüismo. Se estableció así una relación bidireccional entre el PL y el contexto social, político, lingüístico, económico e ideológico en el cual se desarrolla su construcción.

A partir de esta nueva conceptualización del PL y debido a este giro que se ha producido a lo largo de los últimos años sobre la investigación en PL, Ben Rafael, Shohamy, Amara y Trumper-Hecht (2006, 9-10) establecieron tres líneas de corte sociológico que se combinan con la dimensión lingüística para el análisis del PL. Los cuatro autores, partiendo de la noción de contexto sociolingüístico planteada por Landry y Bourhis (op.cit), y de la noción de cambio introducida por Cooper y Spolsky (1991) introdujeron los siguientes enfoques de carácter sociológico en sus investigaciones sobre PL:

El primero, de corte Bourdiano, a través del cual se interconecta la estructuración jerarquizada y desigual del campo social con la configuración del PL;
1. En inglés se usa el concepto de top down vs. bottom-up. El primero se refiere a signos producidos por entidades oficiales, y el segundo a los no oficiales. No obstante, ambas categorías son absolutas ya que en la realidad son más complejas puesto que las lenguas que se pueden escribir o no en el PL están en parte limitadas por leyes en función de los espacios donde están presentes. 
El segundo se basa en la teoría de Goffman (1963) y dirige su atención a la relación que existe entre los procesos de auto-representación, inclusiónexclusión en el espacio público, y las acciones y las prácticas lingüísticas. Del mismo modo, analiza a través de qué modelo está establecido, estructurado y organizado el PL;

Y por último, cabe mencionar el enfoque basado en el concepto de goodreasons, planteado por Boudon (2007). Este enfoque interpreta la estructuración y la configuración del PL en términos de los intereses de los propios actores con respecto a lo público. Es decir, plantea el PL como un recurso que se capitaliza con el objetivo de atraer e influir en el receptor. Dicha interpretación tiene que ver también con lo que Kallen (2009) denomina exotismo en el PL.

Lo cierto es que las investigaciones llevadas a cabo por Ben Rafael et al. (op.cit) se basan en una perspectiva sociológica crítica, y particularmente se interesan por las relaciones de poder en tanto que elemento clave en la configuración del PL. Además, los cuatro investigadores parten del contexto de las ciudades de Israel con una clara presencia de otras comunidades lingüísticas y de otro(s) grupo(s) étnico(s) y religioso(s) como el árabe, el musulmán o el cristiano. Este planteamiento crea entonces un puente entre el análisis del PL y la implicación de lenguas minoritarias o minorizadas. Hay que señalar que dicho enfoque empezó a prestar más atención en sus investigaciones posteriores al análisis de los paradigmas ideológicos, políticos, lingüísticos, sociales, religiosos y étnicos que entran en juego en el proceso de construcción del PL. En este nuevo giro, las investigaciones en PL empezaron a examinar el uso de lenguas minoritarias para la configuración del PL, en términos de procesos de revitalización etnolingüística y de estrategias de resistencia al régimen sociolingüístico institucional dominante o hegemónico, tanto en espacios públicos oficiales como en otro tipo de espacios.

Además de estas líneas de investigación de corte sociológico, otro enfoque que se ha destacado en el análisis del PL es el diacrónico. Este enfoque considera que no se podría entender el PL sin explorar la evolución del PL de forma diacrónica. Y una de sus aportaciones es la que propusieron Barni y Bagna (2009) planteando a través de un análisis diacrónico como objetivo principal, descubrir el modelo lingüístico del PL y los signos que lo han ido configurando y sus características. Ambas autoras también insisten en el desarrollo de una macro-descripción del texto en el PL y del régimen de uso de las lenguas. En este régimen de uso se analiza la estructura y organización del PL como proceso histórico, por un lado, y las dinámicas que se aprecian en esos usos, por otro lado. Barni y Bagna (op.cit), plantean por lo tanto no sólo una macro-descripción en los términos que hemos subrayado antes, sino un análisis micro-lingüístico con distintos niveles de procesamiento, tanto de los datos como del propio análisis (ver Pavlenko 2010).

Recientemente la investigación diacrónica ha tomado de forma notable un giro hacia el método cualitativo. En él se destaca la trascendencia de la comunicación multimodal y del análisis semiótico de los elementos escritos y diseñados en el PL (ver Stroud y Mpendukana 2009, Papen 2012). En opinión de Papen $(2012,58)$, el enfoque diacrónico deberíamos combinarlo con un análisis de las dinámicas de cambio, y señala que "linguistic landscapes as indexical of but also shaped by wider processes of social change and urban development". De este modo, los actores sociales implicados en 
las dinámicas sociolingüísticas empezaron a ser un objeto de interés del enfoque diacrónico en el estudio del PL puesto que desempeñan un papel clave en su configuración. Aunque sobre esta cuestión, Ben Rafael et al. $(2006,8)$ afirmaron anteriormente que el PL ilustra una gran variedad de actores como las instituciones públicas, asociaciones, empresas, individuos, que provienen de diversos estratos. Estos actores no necesariamente actúan de manera armónica, y ni siquiera en algunas veces demuestran una cierta coherencia.

En los últimos años, y a partir de mis investigaciones en el contexto marroquí (Moustaoui 2019), he intentado conectar el vínculo de la conformación de lo urbano y lingüístico con el orden económico neoliberal. Los resultados de estas investigaciones apuntan hacia como la urbanización surge en el marco del capitalismo y el neoliberalismo, y de qué manera cuenta con una racionalidad propia, por la que se impone el valor de cambio frente al valor de uso de la ciudad. Además, pude observar como la globalización neoliberal no ha cambiado en el contexto marroquí la lógica que convierte en mercancía el espacio necesario para la vida cotidiana; al contrario, la ha exacerbado. Es en el espacio urbano donde se apela al consumo, dónde se publicitan los productos y también las lenguas. Las ciudades y las nuevas zonas urbanas híbridas y de contacto entran en competencia para atraer a consumidores, turismo, inversión y retener a sus habitantes. Por lo tanto, según he mostrado, Marruecos no es ajeno a este tipo de fenómenos ya que está viviendo un proceso de urbanización a través del cual el espacio público ha cobrado especial relevancia en la economía local y multinacional. Fundamentalmente, descubrí cómo estos fenómenos han generado nuevas prácticas y nueva regimentación de las lenguas en el espacio a través de un cierto empoderamiento de las lenguas locales, principalmente el árabe marroquí - variedad lingüística históricamente infravalorada y subordinada-.

He podido analizar, de este modo, cómo en estas nuevas condiciones emerge la visibilidad del árabe marroquí en el espacio público, a raíz no solo del actual contexto económico neoliberal marroquí, sino también de la llamada "Primavera Árabe". Estas nuevas prácticas lingüísticas en árabe marroquí han conllevado cambios en la conciencia lingüística y nuevas actitudes de la sociedad hacia esta lengua, que derivaron en su acomodación como un recurso lingüístico bien valorado en el espacio público y en otros espacios de comunicación.

A partir de esta breve contextualización, corroboro que el espacio es un producto social, económico, político, interactivo y abierto para crear nuevas relaciones sociales y nuevos órdenes, lo cual garantiza libertad y autonomía en su construcción social, política y lingüística como veremos más adelante. En la siguiente sección, se desglosan pues las principales categorías que fueron consideradas en el análisis, haciendo especial hincapié en los distintos enfoques de estudio del PL.

\section{Principales enfoques en el estudio del PL}

\subsection{Política lingüística $(P o L)$ y $P L$}

Tal como nota Spolsky (2009), la Política Lingüística (PoL de aquí en adelante) se produce en todos los niveles institucionales y espacios, ya que no 
sólo es un instrumento que controla el estado-nación, sino que se elabora en los barrios, en el seno de las familias y por parte de los individuos a los que históricamente la investigación en PoL ha prestado muy poca atención. Por ello, nociones como engaged language policy introducida por Davis (2014) se centra especialmente en la PoL como iniciativas emprendidas por ciudadanos en el seno de sus comunidades lingüísticas y en distintos ámbitos y espacios al margen de las instituciones. En este sentido, el PL es un mecanismo a través del cual se puede llevar a cabo un ejercicio de la política de la lengua desde abajo, lo cual nos ofrece perspectivas amplias para su exploración desde los estudios de la PoL (Shohamy 2006, 2015). En esta misma dirección, Shoham (2015) considera que existen demandas e iniciativas para gestionar los espacios públicos y hacerlos más inclusivos, abiertos e igualitarios. De allí, la línea de investigación propuesta por Shohamy que conecta el estudio del PL con la PoL. La misma autora afirma que muchas políticas lingüísticas utilizan leyes para regular y ejercer un control sobre el uso de las lenguas en los espacios públicos. No obstante, la aparición de una política de la lengua ejercida desde abajo y hacia arriba (bottom-up), donde actores sociales por iniciativas propias intervienen en la gestión lingüística del espacio, suscitó una nueva línea de investigación de interés para la PoL. Este interés se debe al hecho de que se considera el PL como un potente instrumento potencial para la creación de nuevas políticas de la lengua que resisten y transforman negocian al mismo tiempo- otros regímenes sociolingüísticos y su gestión del uso de las lenguas en el espacio. Barni y Bagna $(2015,12)$ señalan que "The relations between LL (linguistic landscape) studies and studies of language policy have thus become central, giving rise to debate about the linguistic management of territories (understood both as political/ territorial entities and as micro-realities, as occurs in linguistic choices in companies (Lüdi 2012))".

La interconexión pues entre la noción de espacio, PL y PoL propuesta por Shohamy $(2009,2015)$ permite profundizar en la comprensión de facto sobre la PoL y extiende nuestras interpretaciones sobre el lenguaje como práctica espacial y acción humana con una dimensión política e ideológica muy transcendental. Además, Shohamy (2015) también considera que se debe enfocar la aplicación del análisis de la política lingüística a un amplio repertorio del PL, tanto el producido por entidades oficiales autorizadas como por las propias comunidades.

\subsection{Lenguas minorizadas y $P L$}

Entender e interpretar las dinámicas del PL en su relación con la desigualdad y minorización, ha llevado a algunos sociolingüistas a abordar la categoría de lenguas minorizadas en el estudio del PL. Trabajos como los de Cenoz y Gorter (2006) se centraron en la relación que se da entre el PL y el contexto sociolingüístico partiendo del caso del euskera en el país vasco. En concreto, ambos autores vieron como el PL refleja el relativo poder y el estatus que tienen algunas lenguas que están presentes en el espacio. Por su parte, los de Marten et al. (2012) apuntan hacia un enfoque de análisis del PL centrado en las lenguas minoritarias o minorizadas, lo cual permite comprender las luchas que existen entre los hablantes y las estructuras de poder, y en última instancia, empoderaría a los grupos minorizados y daría vitalidad a sus lenguas. El hecho de incluir la noción de lenguas en situación de minorización y subordinación en el análisis del PL, ha podido ayudar en cierta manera a entender la distinción que 
podría existir en la construcción del PL entre lo local/ mayoritario y por un lado, y lo legítimo/deslegítimo, por otro lado. Del mismo modo, la introducción de la noción de minorización fue clave para comprender las relaciones de poder que se dan en el seno del régimen de uso de las lenguas, y su estatus en el nivel local, regional y nacional y sus respectivas identidades. Aunque posteriormente, investigaciones sobre PL y lenguas minorizadas y sus hablantes empezaron a reconsiderar que las nuevas categorías del PL y sus actores no están relegadas a una posición subordinada, sino más bien deben ser reconsideradas como "lenguas" y "hablantes" legítimos que tienen el propio derecho a participar en la construcción del PL y visibilizar sus prácticas lingüísticas en el espacio público (Marten et al. 2012).

Por otro lado, desde una perspectiva de análisis micro-sociolingüístico y escalar (Blommaert 2013b), tanto el contacto entre las lenguas en el PL independientemente de su estatus, como la simultaneidad y la hibridez lingüística podrían intervenir en enfocar las variedades lingüísticas como un conjunto de recursos que podrían estar en situación de igualdad o jerárquicamente estratificados. En este contexto se producen, en palabras de Blommaert (2013b) una indexicalidad a diferentes escalas y conectadas con diferentes roles de los propios hablantes y distintas funciones a las lenguas. Asimismo, según Blommaert, en este espacio de indexicalidad existen también diferentes escalas de autoridad/legitimidad y de restricciones para participar en la construcción del espacio. El enfoque de análisis del espacio y su PL como fenómeno escalar ha ofrecido nuevos datos e interpretaciones sobre la estratificación lingüística, social y política que se da entre las lenguas y las variedades lingüísticas en la sociedad y espacio donde interactúan y están en contacto (SolerCarbonell 2016).

En mis recientes investigaciones, exploré el concepto de Superdiversidad situado en el estudio PL en la ciudad Madrid, un espacio urbano caracterizado no sólo por una situación de minorización a la que están sometidas algunas comunidades inmigrantes sino también de transnacionlismo. A partir de un análisis del PL en lengua árabe en las zonas de contacto en Madrid, descubrí cómo se produce una marcación del espacio como zona de referencia y de cohesión sociolingüística, a través de la recolocación de las lenguas de la inmigración (Moustaoui Srhir 2018). Según postulo, este fenómeno de marcación espacial tiene que ver en cierta medida con los intentos de desnacionalización del espacio público (Sassen 2003), a través de su etnización, del uso de una nueva semiótica racional (Eco 1976) y también mediante de la formación de nuevas expectativas, objetivos y funciones para las nuevas lenguas recolocadas en el espacio por parte de los actores transnacionales, diferentes a los del Estado. Paralelamente a estos fenómenos, y a partir de mi exploración también de las funciones de la lengua árabe en el barrio de Lavapiés en Madrid (Moustaoui Srhir 2018), he descubierto que, independientemente de que exista escalaridad e indexicalidad lingüística, se genera cierta limitación del espacio de referencia de las lenguas hegemónicas, en este caso, el castellano. Como consecuencia, se produce una transformación de las funciones de los espacios y las esferas de interacción, tanto simbólicas, sociales, como económicas, políticas, e incluso religiosas. Una transformación que podríamos interpretarla como forma y práctica de resistencia a la homogeneización y a la hegemonía del castellano estándar, en cierta medida, en el espacio público de Madrid. 


\subsection{Activismo y PL}

La evolución del estudio del PL ha tomado otro rumbo en los últimos cuatro años buscando conexiones entre el PL y distintos modelos de activismo. Hoy en día existe una línea de investigación y de análisis del PL en el marco del estudio de los movimientos sociales que se centra en el movimiento de indignación en algunos países europeos (ver Martín Rojo 2014), y de las últimas revoluciones y movilizaciones que tuvieron lugar en Oriente Medio y el Norte de África en el marco de lo que se denomina la Primavera Árabe (ver Mehrez 2012, Moustaoui Srhir 2016).

Uno de los ejes centrales de esta nueva línea es el análisis y el estudio de la re-configuración del PL en los espacios sociales y virtuales de protesta social y política. La nueva construcción lingüística del espacio, se aborda y se plantea específicamente como nuevas estrategias y prácticas lingüísticas que forman parte de un nuevo modelo de comunicación social y nuevo régimen de uso de la lengua y su política, que resiste al poder y al orden institucional con el fin de deslegitimarlo, planteando transformaciones y cambios en todos los ámbitos (ver Martín Rojo 2014, Mehrez 2012, Moustaoui Srhir 2016).

Esta nueva línea de investigación explora también el PL como objeto etnográfico, donde la observación participante y el trabajo de campo son claves en el análisis contextual y la interpretación sociolingüística del PL y el espacio. Por lo tanto, el nuevo PL como proceso de re-semiotización es considerado, usando las palabras de Martin Rojo (2015), como una apropiación de los espacios urbanos mediante nuevas prácticas lingüísticas que conforman nuevos paisajes que resisten los regímenes sociolingüísticos hegemónicos y los modelos de gobernanza y gestión de los recursos.

La pertinencia de esta perspectiva se ha puesto de manifiesto en el análisis que había realizado dentro de un proyecto desarrollado en el seno del Grupo de Trabajo sobre movimientos sociales, redes y discursos de la Asociación de Estudios sobre Discurso y Sociedad. En esta línea, analicé el uso del árabe marroquí en el seno de los nuevos movimientos y los discursos emergentes que se pueden identificar en estas demandas de ocupación del espacio en Marruecos y que reclaman usos alternativos de los bienes públicos o proponen modelos alternativos de ciudad y de comunicación. El análisis realizado en el contexto marroquí captura asimismo las nuevas identidades o subjetividades que se constituyen en las ocupaciones del espacio y en las prácticas urbanas de los movimientos sociales en Marruecos (Moustaoui Srhir 2016). Esta perspectiva la ha extendido en el último tiempo a un análisis de los procesos des-territorialización/ reterritorialización y cómo se re-contextualizan las prácticas lingüísticas en árabe marroquí (Moustaoui 2019). En este sentido, he estudiado nuevas prácticas en árabe marroquí que han cobrado un valor en el mercado que le dota de visibilidad y legitimidad en el espacio público. El papel y el valor por tanto que conceden los movimientos sociales al árabe marroquí como recurso lingüístico local y su instrumentalización en la ocupación y construcción de nuevos espacios, no solo es evidencia de un cambio en el régimen lingüístico, sino de otros cambios correlativos en la sociedad, como son el activismo de la juventud urbana, los nuevos procesos de urbanización, el auge de las clases medias y el dinamismo económico y geo-demográfico local. 


\subsection{El Ciberespacio y PL}

En la última década, la importancia de las nuevas tecnologías de la comunicación y las redes sociales en la configuración del medio virtual como espacio de relaciones sociales, suscitó la necesidad de estudiar el PL en los entornos virtuales (Ivkovic y Lotherington 2009). En una excelente revisión de la investigación sobre el PL, Marten et al. $(2012,4)$ señalan que el énfasis en la mayor parte del trabajo sobre PL ha sido el signo como objeto estático. Por ello, los autores sugieren que el estudio del PL no se puede limitar exclusivamente a la variedad de indicios estáticos que sólo se encuentran en el espacio público, real o físico. Para extender así los repertorios de investigación en el PL, surgió lo que se denomina el PL online o virtual (Ver Marten et al 2012). Por su parte, Thorne e Ivkovic (2015, 172) afirman que: "As settings for language contact, digital communication media juxtapose and make publicly visible communication from individuals that represent great diversity in terms of spatial location, social positioning, language-culture background and combinations of semiotic resources made relevant to the communicative actions at hand". El objetivo de este enfoque es observar el PL en el ciberespacio a través de distintos métodos de recogida de datos, desde una documentación y catalogación del corpus hasta una observación etnográfica participante. Además, el análisis del PL virtual permite describir y definir la comunidad lingüística y marcar su estatus sociolingüístico a partir de las relaciones de poder expresadas en las diferentes opciones de uso de la lengua que se dan entre las comunidades que interactúan en el ciberespacio.

\section{El trabajo de campo en el estudio del PL}

Una de las preguntas a las que ha de responder cualquier investigador/a es ¿cuál es el área geográfica más relevante para explorar el PL? ¿Cómo elegir esta área geográfica y en base a qué parámetros y criterios? Gorter (2019) presenta una serie de soluciones para los investigadores en las que propone además de seguir los ejes principales del transporte público, centrarse en una o más calles con mayor actividad comercial, ya que tradicionalmente el uso de las calles comerciales como área de análisis en los estudios de PL ha sido una de las opciones más frecuentes. En este sentido, Gorter (op. cit) sugiere también la necesidad de crear el vínculo con el área geográfica de exploración y el vecindario como espacio de encuesta de PL, siendo la encuesta un análisis apropiado y adecuado para combinar junto con la exploración del signo. Por otro lado, creemos que es normal que los investigadores e investigadores intenten recopilar una amplia y representativa sección de unidades de análisis a partir de una o más áreas geográficas. En este sentido, Backhaus (2007) en referencia a la categoría de "unidad de análisis", y a partir de una reflexión sobre la naturaleza de los "signos" y del "lenguaje de los signos", considera la necesidad de reflexionar sobre si los signos son fijos o no, en la medida en que la construcción del PL nunca es sistemática o arbitraria sino es funcional, dinámica e interactiva.

En lo que se refiere a los nuevos métodos de recogida de corpus introducidos por EPL, se ha señalado la relevancia en primer lugar del uso de la cámara de fotos, combinado en segundo lugar con una etnografía sociolingüística del espacio como objeto semiótico, cosmopolita y multilingüe (ver Blommaert et al. 2005, 2013b, 2015a). Es cierto que el acceso a la materia lingüística y al PL que ofrece el espacio público es una tarea fácil a primera vista, con lo cual 
detectar un dato y sacarle fotos resultaría asimismo accesible. El hecho también de que todo el PL está compuesto por datos semióticos (texto, imagen, gestos, colores, movimientos, sonidos) expuestos en espacios donde regularmente no existe cierto control o restricción sobre el uso de la cámara como es la calle, edificios, centros comerciales, etc., nos ayudaría en cierto modo a que nuestro proceso de recogida de datos fuese rápido y abundante en términos, tanto cuantitativos como cualitativos. Sin embargo, a veces no resulta tan fácil desarrollar el trabajo de campo debido a que los agentes implicados en la construcción del PL y las personas en general tienen cierto miedo y reticencia hacia el uso de la cámara de fotos o por lo menos hay cierta restricción en la exploración y observación del espacio. Por este motivo, se requiere pedir en muchos casos permiso, sobre todo cuando se trata de un signo presente en un edificio público como es la administración u otros espacios como las estaciones de tren, las escuelas, los institutos, etc. o incluso en espacios privados.

He de señalar también que el proceso de recogida del corpus no ha de ser sistemático debido a que la construcción del PL tampoco es sistemática, pero sí es dinámica y va cambiando según los acontecimientos, las circunstancias sociales, económicas y políticas del propio espacio como micro-objeto, de las ciudades como entorno urbano y del país en general como espacio geográfico.

En el proceso de recogida de datos también hay que tomar en consideración la diversidad en el repertorio de espacios y tener un conocimiento social, geo-demográfico, económico y urbano del espacio objeto de estudio, para poder desarrollar posteriormente un análisis crítico del contexto que nos ayudaría a procesar, interpretar y examinar nuestros datos en profundidad.

Por otro lado, la recopilación de datos semióticos de manera aislada no tendría cierta legitimidad y rigor sino estuviese combinada y acompañada de una observación etnográfica del espacio y su PL. En este sentido, Blommaert (2015a) como defensor del método ELLA (Ethnographic Linguistic Landscape Analsis), parte de la premisa de que el signo tiene un pasado, un presente y un futuro y unos actores que lo construyen. Esta triple dimensión y de ejes del PL, tanto en el tiempo como en el espacio, en opinión de Blommaert y Maly (op.cit., 194), lo convierte en un proyecto etnográfico por excelencia. La etnografía por lo tanto nos permitiría descubrir cómo los diferentes grupos y sujetos emplean sus recursos semióticos y sus ideologías, por un lado, y cómo organizan sus prácticas y relaciones propias en el espacio, por otro lado. De esta forma, los actores sociales crean y ajustan sus infraestructuras y elementos, adaptando todo esos componentes a sus necesidades comunicativas -en este mismo espacio-, en el que se producen todo tipo de interacciones.

Finalmente, los investigadores e investigadoras en PL deben prestar mucha atención a sus imágenes como datos y también como parte de sus publicaciones, del mismo modo, deberían también reconsiderar las condiciones en las que se producen sus imágenes y los efectos que puedan tener posteriormente.

\section{Nuevos desafíos y direcciones en el estudio del PL}

En noviembre de 2015, se publicó el primer número de la revista Linguistic Landscape: An International Journal, editado por John Benjamins, con un 
equipo editorial compuesto por los especialistas en la temática. Barni y Bagna (2015) en la introducción a este primer número de la revista, señalan que el estudio del PL no se limita únicamente a describir las variedades lingüísticas presentes en ella, sino que implica doble acción. Por un lado, contextualizar el análisis, ampliándolo para abarcar los actores que dan forma o utilizan el paisaje. Y por otro lado, examinar cuáles son los factores que han contribuido a su formación a lo largo del tiempo, abogando por una interdisciplinariedad en el estudio del objeto. Además, según las dos autoras las diferentes metodologías de investigación deben ser utilizadas en función de los objetivos de investigación.

Shohamy (2015), por su parte en el mismo número y continuando en su enfoque que conecta la PoL y el signo, reclama que teorizar las lenguas en los espacios en un marco amplio de estudio e investigación del PL, consiste en tomar en consideración múltiples componentes que van más allá de los textos escritos en los espacios públicos. Estos componentes deberían incluir, entre otros; elementos visuales, sonidos, movimientos, gestos, consideraciones histórico-políticas, la ubicación del propio paisaje, y los actores que lo conforman y configuran. Todo enmarcado y moldeado en la teoría del espacio tal como la concibe Lefebvre (1991). Por su parte, Ben-Rafael y Ben-Rafael $(2015,19)$ enmarcan su análisis del PL en entornos urbanos, caracterizados también por una estructura del caos y los efectos sociales y económicos de la globalización. Ambos autores citan el considerable número de trabajos que se centran actualmente en el análisis del fenómeno de la gentrificación de los entornos urbanos. Los mismos autores señalan que dichos espacios están experimentando cada vez más un conjunto de desarrollos socio-económicos en un contexto de post-industrialización y cambios demográficos fuertes. Se producen por tanto una serie de transformaciones que son el resultado de la movilidad y la presencia de poblaciones cada vez más multilingües y globales o fenómenos producidos por la turistificación. Estos hechos generan un aumento de distintas formas de desigualdad socioeconómica y polarización de los ingresos. Estas nuevas realidades influyen de manera directa en la construcción del espacio y sus paisajes lingüísticos, lo cual invita a analizarlos y prestar más atención a estos nuevos fenómenos.

La publicación de este primer número generó también un intenso debate y una discusión donde la pregunta que planteaba Blommaert (2015a) en otro artículo no publicado en la mencionada revista, era si los estudios sobre PL han de seguir la tradición iniciada y continuada a lo largo de dos décadas. La otra pregunta que planteaba Blommaert (2015a) era si consideramos esta tradición como un canon científico para todos los trabajos, y hasta qué punto habría que seguir una línea que aplicaría nuevos métodos de investigación que rompiesen con dicha tradición (ver Blommaert (2015a) y Blommaert y May (2015). El mismo autor, manifiesta asimismo su disconformidad debido a la ausencia a cualquier mención al enfoque crítico de corte etnográfico - propulsado por el propio Balommaert-, en la mencionada introducción realizada por Barni y Bagna (2015) a este primer número de la revista Linguistic Landscape. Un enfoque etnográfico que ha reclamado y sigue reclamando Blommaert $(2015,2)$ en el que considera que:

Signs are seen as traces of multimodal communicative practices within a sociopolitically structured field which is historically configured [...]. Ethnography is intrinsically historicizing, because any form of effectively performed (and ethnographically monitored) communicative practice can only be made meaningful because of its (Bakhtinian) histories of production and uptake by nonrandomly positioned actors. 
En opinión de Blommaert y Maly $(2015,194)$, el PL es un proyecto etnográfico e histórico. En este proyecto, los signos se consideran índices sobre el tipo de relaciones sociales, intereses y prácticas, y que están dispersados en un campo que está lleno de normas/régimen —en otras palabras del propio Blommaert- que no se solapan. Unas reglas de regimentación que no tienen que ver sólo con el uso de la lengua, sino también con los patrones de conducta, las actitudes, la identidad y legítimas formas de pertenencia de los sujetos que interactúan en el espacio. Todas estas normas pertenecen a diferentes órdenes y operan en distintas escalas y dentro de múltiples historicidades. El PL según Blommaert y Mal (op.cit) se convierte pues en un fenómeno complejo de carácter social que se puede interpretar mediante un análisis de los signos en el espacio público. Del mismo modo, Blommaert (2013b, 2015) parte en su análisis del concepto de la complejidad del sistema sociolingüístico. Este sistema según el mismo autor se caracteriza por la ausencia de una uniformidad y por su carácter policéntrico, donde existen fuerzas internas y externas de cambio perpetuo, que operan de forma simultánea sobre una serie de relaciones inter-mutuas en el espacio y su construcción lingüístico-histórico-discursiva (Blommaert 2013b,10-11). La complejidad del sistema sociolingüístico exige por lo tanto - según propone Blommaert (2013b, 28)-, un Nexus analysis; una especie de intersección de tres nociones y categorías en un tiempo y espacio reales: i) la noción de discourse in place, ii) interaction order, y iii) historical body (Scollon y Scollon 2004 , 19). Con lo cual, el espacio no se debe imaginar como un fondo neutral sino como un objeto clave en unos procesos sociolingüísticos muy complejos.

\section{Conclusiones}

En este artículo hemos podido ver cómo desde sus inicios, los EPL se centraron en analizar la estructura y organización de uso de las lenguas en el espacio y qué dinámicas se aprecian en esos usos, por un lado, y en examinar, por otro lado, el reparto de las lenguas en el paisaje lingüístico en sus distintos repertorios, combinado con una micro-descripción del texto escrito en el PL. Asimismo, se ha considerado los modos en que la sociolingüística urbana pudo demostrar en los últimos años que la ciudad y el espacio urbano operan como mecanismos de homogeneización de la identidad urbana, al mismo tiempo ambos operan también como índices de diferenciación individual y grupal, donde los hablantes desarrollan una serie de prácticas lingüísticas múltiples en función de los contextos de socialización en los que se ven inmersos.

Mi trabajo sobre PL y las dinámicas de cambio en el contexto marroquí demuestra también el papel de la agencia de los hablantes en la transformación de los regímenes lingüísticos mediante la construcción del espacio.

El esbozo que he presentado revela además que la investigación sobre PL se ha caracterizado por una diversidad de enfoques teórico-metodológicos y miradas sobre el objeto de estudio. Y ante la potencialidad y crecimiento de la investigación sobre el PL, emergen cada vez más nuevas miradas donde existe un consenso sobre las complejidades que se pueden encontrar a la hora de abordar el PL. Igualmente, hemos visto en los apartados anteriores que los repertorios del PL son cada vez más amplios en términos de lenguas, espacios y actores que entran en escena. Todos estos elementos son trascendentales para una comprensión más profunda de las sociedades y de la interacción humana en el espacio, tanto el físico-real como el virtual. 
En el artículo hemos podido comprobar también cómo los diferentes enfoques teóricos y las herramientas metodológicas han ido evolucionando con el fin de saciar diferentes objetivos de investigación, describir aspectos específicos del PL e interpretar y comprender el espacio, sea público o privado. Del mismo modo, los enfoques recientes que hemos identificado, reflejan que el estudio del PL no significa limitarse a contar y describir las lenguas presentes en él, sino que implica contextualizar el análisis y combinar sus métodos a través de una etnografía multi-situada e historicista.

Por otro lado, creemos que la relevancia social del tema en este momento concreto de crisis como la de los refugiados, las políticas que cuestionan el derecho al asilo, la desunión de históricas zonas de alianza como la UE con en el Brexit, el fascismo universal o la cuestión del terrorismo y políticas de securitización han generado la necesidad de analizar estas nuevas realidades y de tomar posición en el estudio del PL (Martín Rojo y Moustaoui Srhir 2016). De este modo, estamos asistiendo a un incremento de los métodos y herramientas de análisis para abarcar a los nuevos actores que modelan o utilizan el paisaje, y nuevos factores y contextos contribuyen en su construcción y configuración.

A partir de estas constataciones, reconsideramos que el PL es un excelente campo en desarrollo con una pluralidad de enfoques y multiplicidad de campos de estudio, lo cual genera:

» diversidad de objetos de estudio y contenidos temáticos;

" datos y corpus cada vez más amplios en términos de campo y género discursivo;

» multiplicidad de componentes presentes en el espacio que van más allá de los textos escritos en los espacios públicos (signo, cuerpo, sonidos, icono...)

» necesidad de una investigación/ acción con el propósito de visibilizar y al mismo tiempo de denunciar las violencias lingüísticas que se manifiestan en los paisaje lingüísticos;

" rápida incorporación de la tecnología al servicio del PL con el propósito de elaborar nuevas herramientas digitales interactivas e innovadoras para mapear las lenguas y visibilizar los resultados.

Todas estas dinámicas y transformaciones que ha ido experimentando el campo de investigación en PL plantean la necesidad de un debate metodológico interdisciplinar con una serie de desafíos en la exploración de la relación de la lengua con los distintos espacios y categorías espaciales en los cuales se usa y se instrumentaliza.

Por último, la complejidad cada vez más en aumento de los modelos y sistemas semióticos de interacción humana requiere incorporar de forma más inmediata nuevos conceptos y herramientas de análisis sociolingüístico y multimodal del signo. Los EPL pueden ser un principal foco de investigación capaz de explorar nuevos abordajes metodológicos interdisciplinarios sobre el signo, algunos de los cuales se han introducido en este artículo, que nos permiten describir y comprender realidades sociolingüísticas cada vez más complejas en una moderna tardía impregnada cada vez más de las lógicas neoliberales. 


\section{Q Bibliografía}

" Aladjem, Ruthi y Bibiana Jou. 2016. "The Linguistic Landscape as a Learning Space for Contextual Language Learning”. Journal of Learning Spaces 5(2): 66-70.

" Barni, Monica y Carla Bagna. 2015. “The critical turn in LL. New methodologies and new items in LL". Linguistic Landscape 1/2: 6-18.

" Barni, Monica y Carla Bagna. 2009. "A mapping technique and the linguistic landscape". En Linguistic Landscape: Expanding the Scenery, editado por Elana Shohamy y Durk Gorter. 126-140. New York: Routledge.

» Ben-Rafael, Elieser y Miriam Ben-Rafael. 2015. "Linguistic landscapes in an era of multiple globalizations”. Linguistic Landscape 1/2: 19-37.

»Ben-Rafael, Elieser, Shohamy, Elana, Hasan amara, Muhammed, y Trumper-hecht, Nira. 2006. "Linguistic landscape as symbolic construction of the public space: The case of Israel”. International Journal of Multilingualism 3 (1): 7-30.

"Ben-Rafael, Elieser; Elana Shohamy y Monica Barni. 2010. "Introduction: An approach to an "ordered disorder". En Linguistic Landscape: Expanding the Scenery, editado por Elana Shohamy y Durk Gorter, xi-xxviii. Bristol: Multilingual Matters.

"Blommaert, Jan. 2015. "The conservative turn in Linguistic Landscape Studies. A critical review of the first issue of the journal Linguistic Landscape 1/1-2" fecha de consulta 20 de marzo de 2018. https://www.academia.edu/20057603/The_conservative_turn_in_ Linguistic_Landscape_Studies

" Blommaert, Jan. 2013a. "The Sociolinguistics of Writing”. Journal of Sociolinguistics 17/4: 440-459.

" Blommaert, Jan. 2013b. Ethnography, Superdiversity and Linguistic Landscapes. Bristol: Multilingual Matters.

» Blommaert, Jan. 2010. The Sociolinguistics of Globalization. Cambridge: Cambridge University Press.

"Blommaert, Jan y Ico Maly. 2015. "Ethnographic linguistic landscape analysis and social change”. En Language \& Superdiversity, editado por Jan Blommaert, Ben Rampton, Karel Arnaut y Massimiliano Spotti, 191-211. London \& New York: Routledge.

»Blommaert, Jan, Jim Collins y Steph Slembrouk. 2005. “Spaces of multilingualism”. Language \& Communication 25: 197-216.

"Boudon, Raymon. 2007. The origin of values: Sociology and philosophy of beliefs. New Brunswick, NY: Transactions Publishers.

》 Bogatto, François y Christine Helot. 2010. "Linguistic landscape and language diversity in Strasbourg: The "Quartier Gare”. En Linguistic Landscape in the City, editado por Elana Shohamy, Elieser Ben-Rafael y Monica Barni, 275-291. Bristol: Multilingual Matters.

" Brown, Kara. 2012. "The Linguistic Landscape of Educational Spaces: Language Revitalization and Schools in Southeastern Estonia". En Minority Languages in the Linguistic Landscape, editado por Durk Gorter, Heiko Marten y Luk Van Mensel, 281-298. London: Palgrave McMillan.

" Brown, Kara. 2005. "Estonian Schoolscapes and the Marginalization of Regional Identity in Education". European Education 37: 78-89.

» Castillo Lluch, Mónica y Daniel Sáez Rivera. 2013. “Introducción”. Revista Internacional 
de Lingüística Iberoamericana, vol. XI, 1/21: 9-22.

» Castillo Lluch, Mónica y Daniel Sáez Rivera. 2011. “Introducción al paisaje lingüístico de Madrid”. Lengua y Migración 3: 73-88.

»Eco, Umberto. 1976. A Theory of Semiotics. Bloomington: Indiana University Press.

» Gorter, Durk y Jasone Cenoz. 2015. “The linguistic landscapes inside multilingual schools". En Challenges for language education and policy: Making space for people, editado por Bernard Spolsky, Michal Tannenbaum y Ofra Inbar, 151-169. New York: Routledge.

»Cenoz, Jasone y Durk Gorter. 2006. “Linguistic landscape and minority languages”. International Journal of Multilingualism 3 (1): 67-80.

»Cooper, Robert y Bernard Spolsky. 1991. The languages ofJerusalem. Oxford: Clerendon.

» Coupland, Nicolas y Peter Garret. 2010. “Linguistic landscapes, discursive frames and metacultural performance: the case of Welsh Patagonia". International Journal of Sociology of Language 205: 7-36.

» Gorter, Durk. 2019. “Methods and Techniques for Linguistic Landscape Research: About Definitions, Core Issues and Technological Innovations”. En Expanding the Linguistic Landscape: Multilingualism, Language Policy and the Use of Space as a Semiotic Resource, editado por Martin Pütz y Neele Mundt, 38-57. Bristol: Multilingual Matters.

» Gorter, Durk. 2006. "Introduction: The study of linguistic landscape as a new approach to multilingualism”. International Journal of Multilingualism 3(1): 1-6.

» Davis, Kathryn. 2014. “Engaged language policy and practice”. Language Policy 13(4): 83-100.

» Gorter, Durk, ed. 2006. Linguistic Landscape. A New Approach to Multilingualism. Bristol: Multilingual Matters.

» Heller, Monica. 2010. "Language as resource in the globalised new economy”. En The Handbook of Language and Globalization, editado por Nicolas Coupland, 349-365. Blackwell: Blackwell Publishing.

» Heller, Monica. 2005. “Une approche sociolinguistique à l'urbanité”. Revue de l'Université de Moncton 36/1: 321-346.

» Heller, Monica y Jossiane Boutet. 2006. "Vers de nouvelles formes de pouvoir langagier? Langue(s) et identité dans la nouvelle économie”. Langage et Société 118 : 5-16.

» Ivkovic, Dejan y Heather Lotherington. 2009. “Multilingualism in cyberspace: Conceptualising the virtual linguistic landscape". International Journal of Multilingualism 6 (1): $17-36$.

» Javorski, Adam. 2010. "Linguistic landscapes on postcards. Tourist mediation and the sociolinguistic communities of contact”. Sociolinguistic Studies (4) 3: 469-594.

» Kallen, Jeffrey. 2009. "Tourism and representation in the Irish linguistic landscape", En Linguistic Landscape: Expanding the Scenery, editado por Elana Shohamy y Durk Gorter, 270-284. New York: Routledge.

"Landry, Rodrigue y Richard Bourhis. 1997. "Linguistic landscape and ethnolinguistic vitality: An empirical study”. Journal of Language and Social Psychology 16: 23-49.

»Lefevbre, Henri. 1991. The production of space. Oxford: Blaxkwell Publishing.

» Leeman, Jennifer y Gabrielle Modan. 2009. “Commodified language in Chinatown: A contextualized approach to linguistic landscape”. Journal of Sociolinguistics 13: 332-362.

» Leeman, Jennifer y Gabrielle Modan .2010. "Selling the city: Language, ethnicity and commodified space". En Linguistic Landscape in the City, editado por Elana Shohamy, 
Elieser Ben-Rafael y Monica Barni, 182-198. Bristol: Multilingual Matters.

" Lillis, Teresa y Carolyn Mackinney. 2013. "The Sociolinguistics of Writing in a global context: Objects, lenses, consequences”. Journal of Sociolinguistics 17/4: 415-439.

" Lüdi, Georges, Katharina Höchle y Patchareerart Yanaprasart. 2010. "Patterns of language in polyglossic urban areas and multilingual regions and institutions: a Swiss case study". International Journal of Sociology of Language 205: 55-78.

» Marten, Heiko, Luk Van mansel y Durk Gorter. 2012. "Introduction. Studying Minority Languages in the Languistic Landscape". En Minority Languages in the Linguistic Landscape, editado por Heiko Marten, Luk Van Mansel y Durk Gorter, 1-15. Hampshire: Palgrave Macmillan.

" Martin Rojo, Luisa. 2014. "Taking over the Square: The Role of Linguistic Practices in Contesting Public Spaces". Journal of Language \& Politics 83: 1-22.

" Martin Rojo, Luisa. y Adil Moustaoui Srhir. 2016. "Nuevos desafíos en los estudios del PL”. Trabajo presentado en el Taller Internacional Paisaje Lingüístico de la migración. Roma, La Sapienza Universitá di Roma, 24-26 de octubre.

» Mehrez, Samia, ed. 2012. Translating Egypt's Revolution. The Language of Tahrir. Cairo/ New York: The American University in Cairo Press.

» Moustaoui, Adil. 2019. "Transforming urban public space: Linguistic landscape and new linguistic practices in Moroccan Arabic”. Linguistic landscape 5 (1): 80-102.

" Moustaoui Srhir, Adil. 2018. "Recontextualización sociolingüística y Superdiversidad. El árabe en el paisaje lingüístico del barrio de Lavapiés en Madrid”. Lingue e Linguaggi 25: 197-225.

" Moustaoui Srhir, Adil. 2016. "Resisitiendo el regimen sociolingüístico institucional: Nuevas prácticas lingüísticas como estrategias de comunicación en el seno del Movimiento 20 de Febrero en Marruecos". Ediso working papers 1: 1-15.

"Moustaoui Srhir, Adil. 2013. "Nueva economía y dinámicas de cambio sociolingüístico en el paisaje lingüístico: el caso del árabe en Madrid”. Revista Internacional de Lingüística Iberoamericana, vol. XI. 1/21: 89-108.

"Moustaoui Srhir, Adil y García Agustín, Oscar. 2015. “Espacios urbanos en transformación: comunicación y discursos de resistencia". Trabajo presentado en el II Simposio de EDiSo Discursos y sociedades en movimiento, Centro de Estudios Sociales, Coimbra, 18-20 de junio.

"Papen, Uta. 2012. “Commercial discourses, gentrification and citizen's protest: The linguistic landscape of Prenzlauer Berg, Berlin”. Journal of Sociolinguistics 16: 56-80.

"Pavlenko, Aneta. 2010. "Linguistic landscape of Kyiv, Ukraine: A diachronic study". En Linguistic Landscape in the City, editado por Elana Shohamy, Elieser Ben-Rafael y Monica Barni, 133-150. Bristol: Multilingual Matters.

"Prego Vázquez, Gabriel y Luz Zas Varela. 2018. “Paisaje lingüístico. Un recurso TIC-TACTEP para el aula”. Lingue e Linguaggi 25: 277-295.

"Sassen, Saskia. 2003. "Globalization or Denationalization". Review of International Political Economy 10 (1): 1-22.

» Soler-Carbonell, Josep. 2016. "Complexity perspectives on linguistic landscapes. A scalar analysis". Linguistic Landscape 2:1: 1-25.

"Shohamy, Elana, 2015. "LL research as expanding language and language policy". Linguistic Landscape 1 (1) 152-171.

"Shohamy, Elana y Durk Gorter, eds. 2009. Linguistic Landscape: Expanding the Scenery. 
New York: Routledge.

" Robertson, Roland. 1995. “Glocalization: time and space and homogeneity-heterogeneity”. En Global Modernities, editado por Mike Featherstone, Schott Lash y Roland Robertson, 1-24. London, Thousand Oaks, New Delhi: Sage Publications.

"Spolsk, Bernard. 2004. Language Policy, Cambridge: Cambridge University Press.

"Spolsky, Bernard y Elana Shohamy. 1999. Languages of Israel: Policy, Ideology and Practice. Clevedon: Multilingual Matters.

» Stroud, Christopher y Sibonile Mpendunkana. 2009. “Towards a material ethnography of linguistic landscape: Multilingualism, mobility and space in a South African township". Journal of Sociolinguistics 13 (3): 393-386.

"Van Mensel, Luck, Mieke Vandenbroucke y Robert Blackwood. 2016. "Linguistic Landscapes". En The Oxford Handbook of Language and Society, editado por Ofelia García, Max Spotti y Nelson Flores, 423-450. Oxford: Oxford University Press. 